\title{
Closed Drainage versus Non-Drainage for Single-Level Lumbar Disc Surgery: Relationship between Epidural Hematoma and Fibrosis
}

\author{
Kadir Kotil \\ Department of Neurosurgery, Istanbul Arel University, Istanbul, Turkey
}

Study Design: A prospective clinical series with prospectively collected data.

Purpose: The efficacy of using closed suction drains (CSD) after single-level lumbar disc surgery was evaluated. Postoperative CSD are regularly fitted to prevent postoperative epidural hematomas (EH) after multilevel lumbar decompression, although it remains unclear whether CSD also reduces postoperative EH following single-level lumbar disc surgery.

Overview of Literature: Few articles have addressed the clinical outcome in patients with single-level lumbar disc disease who were treated by two different operative methods (with and without drainage).

Methods: Between 2012 and 2014, 115 patients with a single level discectomy underwent two surgical procedures: with CSD (group A, 60 cases) and without CSD (group B, 55 cases). There were no significant differences in age, sex, segment level, herniation type, or disease duration between the groups. Wound infection, $\mathrm{EH}$, and epidural fibrosis (EF) were evaluated by magnetic resonance imaging. Pain intensity was evaluated using the visual analog scale (VAS) and Oswestry disability index (ODI). Reduction in analgesic treatment and patient satisfaction were also recorded.

Results: The overall rate of postoperative EH was $5 \%$ and $16.3 \%$ in group A and B, respectively, whereas the rate of postoperative EF was $11.6 \%$ in group $A$ and $21.8 \%$ in group $B$. The postoperative VAS score was 0.32 (standard deviation [SD], 0.45 ) for group $A$ and 2.62 (SD, 06.9) for group $B$, whereas $0 D I$ was 9.11 (SD, 0.68) and 8.23 (SD, 0.78) for group A and and group B, respectively, with no significant differences observed.

Conclusions: In patients operated on by unilateral, single-level lumbar disc surgery, the use of suction CSD into the operation site results in lower levels of EH and EF radiologically, thereby providing a better clinical outcome.

Keywords: Spine; Hematoma; Fibrosis; Drainage; Back pain

\section{Introduction}

Postoperative epidural hematomas (EH) have been reported as "very frequent" when radiologic investigation of the postoperative lumbar spine is performed [1]. Postop- erative lumbar epidural fibrosis (EF) is the formation of scar tissue over the dura mater following EH [1-9]. EHs on the first postoperative day of lumbar disc surgery were reported to occur as frequently as $86 \%$ after using magnetic resonance imaging (MRI) [9]. In addition, symp-

Received Mar 4, 2016; Revised May 23, 2016; Accepted May 24, 2016

Corresponding author: Kadir Kotil

Department of Neurosurgery, Istanbul Arel University, Tepekent, Istanbul, Turkey

Tel: +90-216-360-12-26, Fax: +90-216-302-03-91, E-mail: kadirkotil@gmail.com 
tomatic EH is common after lumbar disc surgery [9-11]. Symptomatic patients demonstrate a typical progression from sharp operi-surgical pain to neurological deficits. Little is known about what differentiates symptomatic and asymptomatic patients. The quoted incidence of postoperative symptomatic EH occurring after all spinal procedures requiring surgical intervention ranges from $1 \%$ to $2.9 \%[1,6,9]$. MRI measurements of hematoma size or mass may correlate with postoperative symptoms. In addition, compression resulting from postoperative $\mathrm{EH}$ of the nerve roots is a well-known complication of lumbar disc surgery that may lead to intractable back and neurological deficit in acute postoperative periods. Moreover, one other late complication is EF. Kotilainen et al. [11] reported that the amount of epidural scar tissue at 6 months postoperatively significantly correlated with the extent of hemorrhagic changes detected in MRI scans on the first postoperative day. Various methods have been used to prevent significant fibrosis postoperatively with variable degrees of success. Postoperative lumbar closed suction drains (CSD) has traditionally been used to prevent postoperative hematomas. In addition, a hematoma also provides an excellent culture medium for infections. The evacuation of an $\mathrm{EH}$ is considered advisable in order to prevent both postoperative pain, infections, and EF $[3,7,9]$. CSD is believed to be effective in preventing these complications in spine surgery [1]. However, the current spine literature has not shown any advantage for the use of CSDs in EF $[7,12]$. In this study, it was proposed to determine and compare the incidence and size of $\mathrm{EH}$, and late scar formation, by means of MRI techniques in patients in group $\mathrm{A}$ and $\mathrm{B}$.

\section{Materials and Methods}

In this study, 115 consecutive patients who underwent single-level lumbar microdiscectomy during a 2-year period (2012 and 2014) were reviewed. A total of 115 patients with single-level discectomy underwent 2 surgical procedures using either CSD (group A, 60 cases) or without CSD (group B, 55 cases). In group A, there were 28 males and 32 females with an average age of 42.3 years (range, 28-67 years) including 6 cases at the level of L2-3, 9 cases at L3-4, 21 cases at L4-5, and 23 cases at L5-S1. In group B, there were 29 males and 26 females with an average age of 41.8 years (range, 27-70 years); including 4 cases at the level of L2-3, 8 cases at L3-4, 20 cases at L4-5, and 23 cases at L5-S1. There were no significant differences in age, sex, segment level, herniation type, or disease duration between the 2 groups $(p>0.05)$. Wound infection, EH, and EF were evaluated by both early and late MRI with contrast enhancement although we are unable to collect data about the classification of the herniation. Pain intensity was evaluated using visual analog scale (VAS) and Oswestry disability index (ODI). Reduction of analgesic treatment and patient satisfaction were also recorded. All surgery techniques were the same and single-level microdiscectomy was performed with a hemipartial laminectomy. All patients were operated on in the prone position under general anesthesia, and systemic prophylactic antibiotic therapy with cefazolin was administered intravenously in a dose of $2 \mathrm{~g}$ at 1 hour before surgery and $1 \mathrm{~g}$ every 8 hours postoperatively, to a maximum of three doses. Those who underwent multilevel disc surgery or who were considered complicated cases (such as dural tearing or sequestrectomy) were not included in this study. Epidural hemostasis was achieved with temporary compression and bipolar coagulation. In group A, a 14-French silicone CSD was inserted into the surgical area (Fig. 1). The CSDs were maintained for 12-18 hours postoperatively (when sediment forms the CSD is then removed). After the CSD was withdrawn, the total CSD was noted, and the patient was mobilized. All CSD were used with mild suction pressure (half negative). Postoperatively, the CSD was removed when the amount of bleeding did not exceed $10 \pm 3 \mathrm{~mL}$ per day. All patients were discharged from hospital between 18 and 24 hours postoperatively.

MRI examinations were performed on all patients on
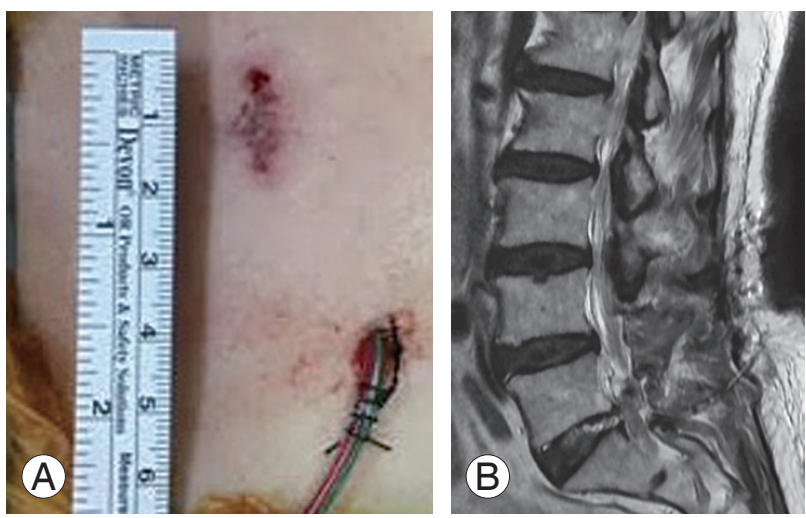

Fig. 1. In group A, a 14-French silicone closed suction drain was inserted into the epidural space (A), a sagittal magnetic resonance imaging showing a silicon tube inserted into the epidural space (B) 
postoperative days which included the first day postoperatively as well as 6 months and 12 months postoperatively. We performed the MRI in all discectomy patients who only had a single-level disc herniation using a selection of MRI machines. Follow-up MRI scans were performed 1 day postoperatively (without contrast), and at 6 and 12 months (with contrast) postoperatively (mean follow-up MRI time, 9.2 months). MRI examinations followed the same protocol at each clinical site, as follows (Fig. 2): (1) before contrast administration, two-dimensional axial T1-weighted spin-echo and T2-weighted turbo spin-echo sequences and sagittal T1-weighted spin-echo and T2-weighted spin-echo sequences; (2) administration of $0.1 \mathrm{mmol} / \mathrm{kg}$ gadolinium contrast material via slow intravenous push; and (3) axial and sagittal T1-weighted spin-echo sequences, completed within 15 minutes after administration of contrast material. Visual assessment of the hematoma was performed by a radiologist who was blinded to the patient groups. Postoperative hematoma evaluation with MRI was determined by hematoma grading [1]. The maximum thickness of the $\mathrm{EH}$ was measured on the axial slice and graded as none (<1 mm), minimal (1-1.9 mm), moderate (2-2.9 mm), or prominent $(>3 \mathrm{~mm})$.

\section{EF grading}

The amount of EF was graded on a scale of 0-4 for each quadrant of each imaging slice encompassing the operative level [9]: 0, no sign of a scar to trace scar; 1, 2, 3, and 4, signs of scarring more than to $25 \%, 50 \%, 75 \%$, and $100 \%$, respectively. If the patient had a scar score of 4 in any one of the quadrants then it was defined as an "extensive scar." The whole case was described as having none, mild, moderate, or extensive EF (Fig. 3), MRIs right posterior EF of all grades.

\section{Results}

The mean operating time $50 \pm 5$ minutes and intraoperative blood loss $60 \pm 10 \mathrm{~mL}$ were similar in both groups. There was a significant difference in hematoma formation when the 2 groups were compared. There was no difference in the risk of wound infection as a result of avoiding postoperative closed-suction CSD in these 2 groups of patients. Two patients in group B developed postoperative EHs which caused neurological compromise with both undergoing surgical evacuation (Fig. 2A). Only 2 patients in group $\mathrm{B}$ were operated on due to acute $\mathrm{EH}$, and one of these patients had acute back and leg pain. T2-weighted and gadolinium-enhanced MRI results showed hematoma formation at the epidural space between L4 and L5. Immediately after the MRI, extended unilateral hemilaminectomy and hematoma evacuation were performed. The patient achieved complete neurological recovery. In the second case in group B, the patient was a 71-year-old female who had L5/S1 disc herniation. This patient had comorbidities including osteoporosis and hypertension. At the initial surgery, microdiscectomies were performed and neurological deterioration developed on the day of surgery. Axial and sagittal T2-weighted MRI results showed
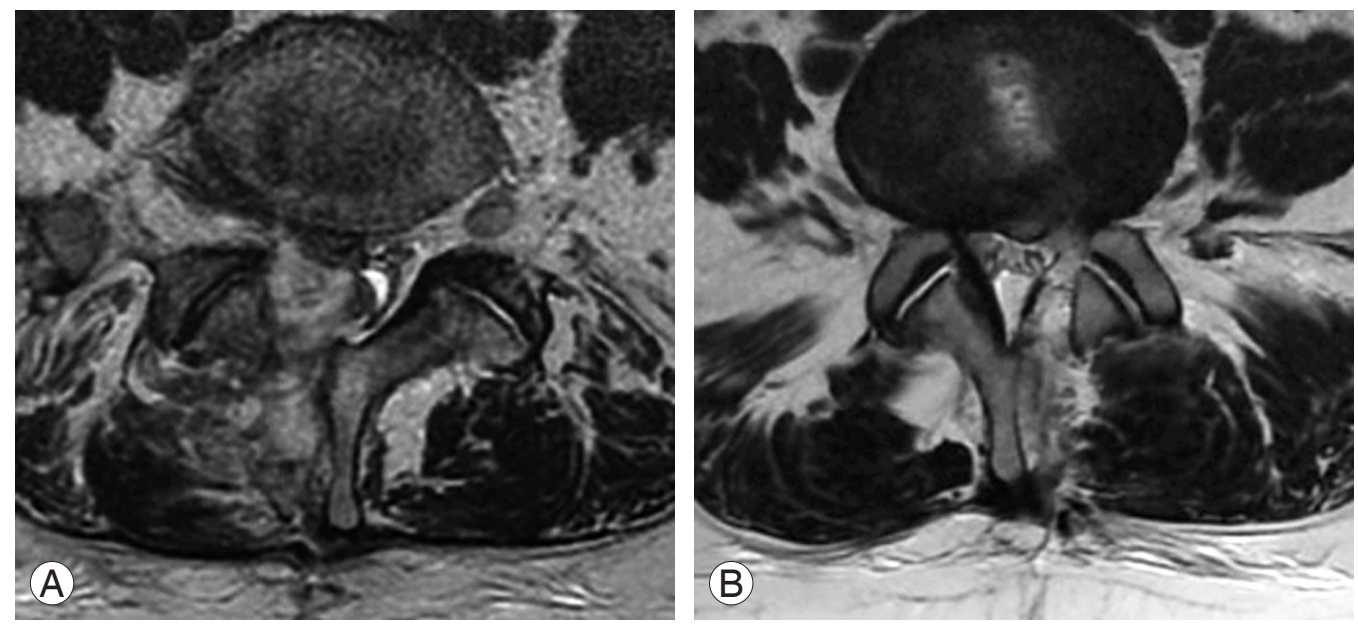

Fig. 2. (A) T2-weighted axial magnetic resonance imaging showing hematoma formation (group B) at the epidural space between $L 4$ and $L 5$, one day postoperative. (B) T2-weighted axial magnetic resonance image shows minimal hematoma formation (group A) at the epidural space. 

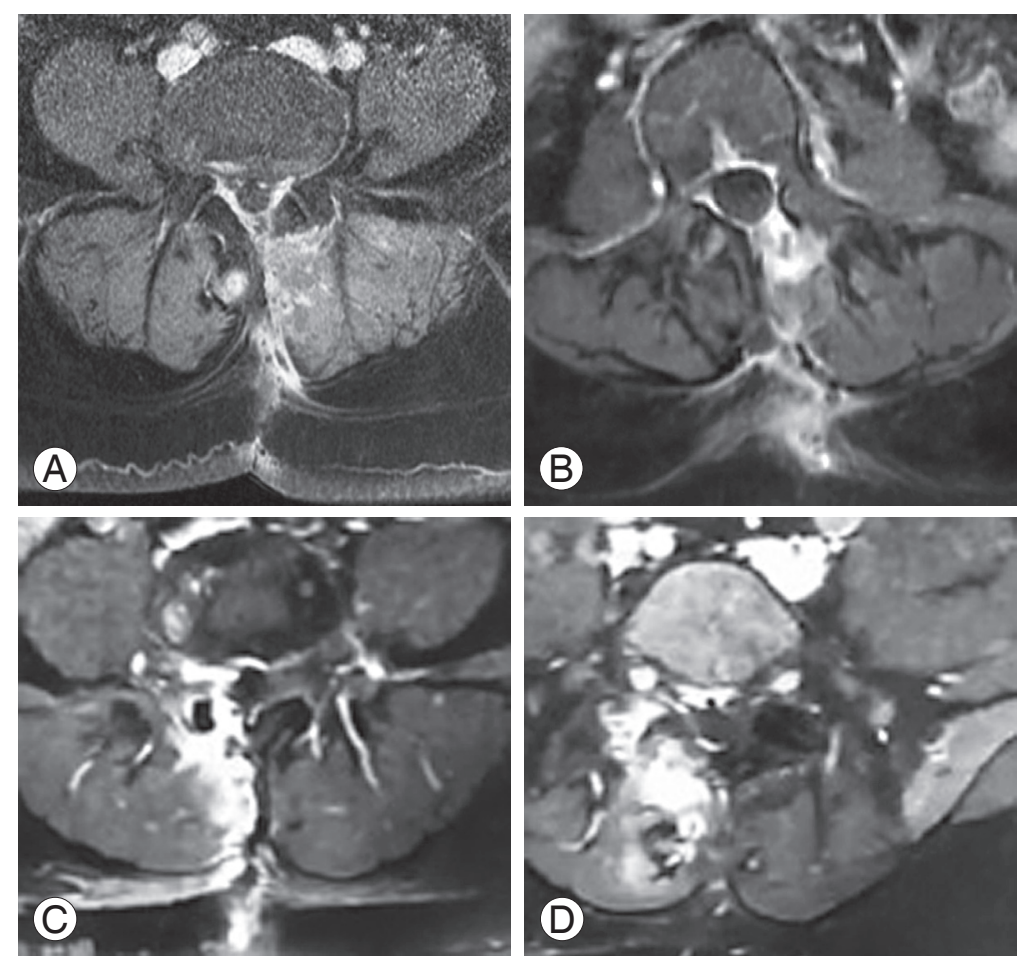

Fig. 3. The grading system of epidural fibrosis with T1 weighted axial magnetic resonance imaging in postoperative period (12 months): (A) grade 1; (B) grade 2; (C) grade 3; and (D) grade 4.

hematoma formation at the epidural space between L5 and S1. On the day of the initial surgery, a hematoma was evacuated using the same approach. The patient achieved complete neurological recovery.

As a result, the overall rate of prominent postoperative EH was $5 \%$ in group A and $16.3 \%$ in group B (Table 1). In addition, the overall rate of severe postoperative EF was $11.6 \%$ in group A and $21.8 \%$ in group B. The distribution of the various degrees of fibrosis (maximum values) in the two groups is shown in Table 2. Postoperative scores for VAS and ODI are given in Table 3. The postoperative VAS score was 0.32 (standard deviation [SD], 0.45) for group A and 2.62 (SD, 0.69) for group B. We did not record the leg VAS score but would have done if we had conducted this study again. Analgesic consumption was stopped or reduced in group A patients (85\%) 6 days postoperatively and in group B patients (72\%) 10 days following the procedure. In the end, overall patient satisfaction for group A patients was 85\% 2 weeks and 15\% 6 months postoperatively, which was the final follow-up. ODI was 9.11 (SD, 0.68) for group A and 8.23 (SD, 0.78) for group B. Significant differences were not observed between the two groups. Both VAS scores and Prolo scale were the same between 6 and 12 months postoperatively (Table 3). In the end, ODI was significantly higher in group A when compared with group B cases $(p=0.053)$. However, the

Table 1. EHs observed on MRI (no. of patients=115)

\begin{tabular}{|c|c|c|c|c|c|c|}
\hline \multirow{2}{*}{ Group } & \multicolumn{4}{|c|}{$\mathrm{EH}$} & \multirow{2}{*}{ Epidural fibrosis } & \multirow{2}{*}{$p$-valu } \\
\hline & None & Minimal & Moderate & Prominent & & \\
\hline Group A (CSD+) & $9(15)$ & 43 (71.6) & $5(8.3)$ & $3(5)$ & $12(9.3)$ & 0.05 \\
\hline Group B (CSD-) & - & $5(9.9)$ & 41 (74.5) & $9(16.3)$ & 28 (50.9) & 0.04 \\
\hline
\end{tabular}

Values are presented as number (\%).

EH, epidural hematoma; MRI, magnetic resonance imaging; CSD, closed suction drain. 
Table 2. EF grading on MRI

\begin{tabular}{lcccccc} 
& No. of patients & & \multicolumn{3}{c}{ EH } \\
\cline { 3 - 6 } Group & $(\mathrm{n}=115)$ & Grade 0 & Grade 1 & Grade 2 & Grade 3 & Grade 4 \\
Group A (CSD+) & 60 & $3(5)$ & $29(48.3)$ & $10(16.6)$ & $11(18.3)$ & $7(11.6)$ \\
Group B (CSD-) & 55 & 0 & $5(9)$ & $18(32.7)$ & $20(36.6)$ & $12(21.8)$ \\
\hline
\end{tabular}

Values are presented as number (\%).

EF, epidural fibrosis; MRI, magnetic resonance imaging; EH, epidural hematoma; CSD, closed suction drain.

Table 3. Both ODI and VAS values of the groups after the surgery

\begin{tabular}{|c|c|c|c|c|c|}
\hline \multirow{2}{*}{ Group } & \multicolumn{2}{|c|}{ VAS lumbar back pain } & \multicolumn{2}{|c|}{ Postoperative ODI (6/12 mo) } & \multirow{2}{*}{$p$-value } \\
\hline & 1 day & $6 \mathrm{mo}$ & $6 \mathrm{mo}$ & $12 \mathrm{mo}$ & \\
\hline Group A & $2(2-5)$ & $1(1-4)$ & $22.6(11-52)$ & 9.11 & 0.05 \\
\hline Group B & $5(2-7)$ & $3(2-5)$ & $28.7(4-51)$ & 8.23 & 0.06 \\
\hline
\end{tabular}

ODI, Oswestry disability index; VAS, visual analog scale.

ODI scores at the final follow-up sessions between 6 and 12 months postoperatively did not show a significant difference.

\section{Statistical analysis}

All statistical analyses were performed on a personal computer. The differences of parameters among the two groups were assessed using a linear by linear association test. The differences of parameters between the two groups were assessed using a $t$-test paired samples test. The level of significance was set at $p=0.05$.

\section{Discussion}

Risk factors for spinal EH after spinal surgery are well known. EH is an acute problem, whilst EF is a late complication following lumbar disc surgery [2-7]. Early postoperative back and leg pain, paresthesias, acute neurologic deficit or cauda equina syndrome, as well as infection can all be caused by EH. Possible mechanisms of recurrent pain caused by $\mathrm{EH}$ include nerve root irritation and entrapment, unilateral or bilateral direct dural sac compression, restriction of nerve root mobility, and epidural fibrotic tissue formation $[3,6,9,13]$. EF is a natural result of normal postoperative recovery, resulting in symptoms caused by adherence to nerve roots $[7,8]$. EF is formed by intense fibrous tissue on the surface of paravertebral muscles and the fibrous layers of the periosteum, and also by invasive postoperative EH. Ross et al. [10] found in a multicentered randomized trial that an increasing amount of scarring led to a significantly increased likelihood of recurrent radicular pain. This association was found only in a small subgroup of 20 patients experiencing recurrent radicular pain after discectomy. A variety of surgical techniques and treatments have been discussed in the literature in terms of reducing EF. Reoperation on scar tissue often produces a poor surgical result and further scarring $[6,8,10,11,13]$. The best treatment for EF is prevention. Minimal invasive surgery with smaller incisions and muscle dissection, intraoperative restricted manipulations and appropriate hemostasis were reported to reduce the amount of EF [13]. In the literature, EF was reported to be reduced by the use of hemostatic agents and anti-inflammatory drugs (methylprednisolon, triamcinolone, prednisolone, ketoprofen, dexamethasone) and by mechanical barriers inserted between the tissue and dura mater as well as the use of synthetic and biological materials $[14,15]$. The clinical use of these agents has not been successful [14]. Fat grafts were applied as an initial treatment to prevent EF after lumbar discectomy. Fat grafts were subcutaneously obtained from the surgical incision site and placed over the dura in order to cover the dura. This fat grafting was reported to have no preventive effects on EF, and eventually caused cauda equina syndrome [14].

CSD has long been been used following extensive spinal surgery. In contrast, the use of closed suction CSD after 
single-level disc surgery remains controversial [12]. However, the study in question looks at a prospective clinical series in comparison to prospectively collected data. As such, this study reviewed a large number of patients undergoing single-level disc surgery in order to evaluate the efficacy of CSD. None of the 115 patients suffered wound infection requiring surgical intervention with the rate of postoperative $\mathrm{EH}$ being $5 \%$ in group $\mathrm{A}$ and $16.3 \%$ in group B. These results suggest that the risk of postoperative $\mathrm{EH}$ increased without the placement of a CSD. In addition, EF is caused by EH, although many cases tend not to lead to symptoms. Low back pain is the most important factor in the early postoperative period for both the surgeon and patient. This is due to back motion being restricted by $\mathrm{EH}$. Radiological investigation shows us that postoperative muscle edema and hematoma are a bigger issue than EF. Mirzai et al. [1] evaluated the efficacy of closed-suction CSD in lumbar disc surgeries. They prospectively randomized 50 (22 with CSD and 28 without CSD) patients into either a "CSD" or "no CSD" group. They also reported that the use of closed-suction CSD did result in hematoma in lumbar disc surgery. Awad et al. [2] analyzed the records of 14,932 patients who underwent spinal surgeries to identify the risk factors of postoperative spinal EH. They showed more than five operative levels, anemia $(<10 \mathrm{~g} / \mathrm{dL})$, and excessive blood loss $(>1 \mathrm{~L})$ increased the risk of $\mathrm{EH}$ formation.

Kanayama et al. [12] reported in their study, that the risk of wound infection and hematomas in single-level lumbar decompression surgery was not influenced by the use of a drain. The use of postoperative wound drainage in patients with a potential risk for epidural bleeding in situations such as multiple-level decompression, instrumentation surgery, anticoagulant therapy, trauma, and tumors or metastases requires additional study. In contrary, we reviewed a large number of patients undergoing single-level disc surgery to evaluate the efficacy of CSD. The rate of postoperative EH was higher in group B. This hypothesis was therefore rejected by our study. Postoperative spinal EH can thus be prevented by CSD.

\section{Conclusions}

This study therefore concludes that, in patients operated on for unilateral, single-level lumbar disc surgery, implantation of suction CSD into the operation site results in lower levels of $\mathrm{EH}$ and EF radiologically, and as such yields a better clinical outcome. Early back comfort is clearly evident in cases who have used CSD. As a result, this study recommends the use of a drain when singlelevel lumbar disc surgery is performed.

\section{Conflict of Interest}

No potential conflict of interest relevant to this article was reported.

\section{References}

1. Mirzai H, Eminoglu M, Orguc S. Are drains useful for lumbar disc surgery? A prospective, randomized clinical study. J Spinal Disord Tech 2006;19:171-7.

2. Awad JN, Kebaish KM, Donigan J, Cohen DB, Kostuik JP. Analysis of the risk factors for the development of post-operative spinal epidural haematoma. J Bone Joint Surg Br 2005;87:1248-52.

3. Gabriel EM, Friedman AH. The failed back surgery syndrome. In: Wilkins RH, Rengachary SS, editors. Neurosurgery. New York: McGraw-Hill; 1996. p.3863-70.

4. Di Lauro L, Poli R, Bortoluzzi M, Marini G. Paresthesias after lumbar disc removal and their relationship to epidural hematoma: report of two cases. J Neurosurg 1982;57:135-6.

5. Brown MD, Brookfield KF. A randomized study of closed wound suction drainage for extensive lumbar spine surgery. Spine (Phila Pa 1976) 2004;29:1066-8.

6. Lawton MT, Porter RW, Heiserman JE, Jacobowitz R, Sonntag VK, Dickman CA. Surgical management of spinal epidural hematoma: relationship between surgical timing and neurological outcome. J Neurosurg 1995;83:1-7.

7. Payne DH, Fischgrund JS, Herkowitz HN, Barry RL, Kurz LT, Montgomery DM. Efficacy of closed wound suction drainage after single-level lumbar laminectomy. J Spinal Disord 1996;9:401-3.

8. Sen O, Gokcel A, Kizilkilic O, et al. The relation between serum levels of osteoprotegerin and postoperative epidural fibrosis in patients who underwent surgery for lumbar disc herniation. Neurol Res 2005; 27:452-5.

9. Mohi Eldin MM, Abdel Razek NM. Epidural fibrosis after lumbar disc surgery: prevention and outcome evaluation. Asian Spine J 2015;9:370-85. 
10. Ross JS, Robertson JT, Frederickson RC, et al. Association between peridural scar and recurrent radicular pain after lumbar discectomy: magnetic resonance evaluation. ADCON-L European Study Group. Neurosurgery 1996;38:855-61.

11. Kotilainen E, Alanen A, Erkintalo M, Helenius H, Valtonen S. Postoperative hematomas after successful lumbar microdiscectomy or percutaneous nucleotomy: a magnetic resonance imaging study. Surg Neurol 1994;41:98-105.

12. Kanayama M, Oha F, Togawa D, Shigenobu K, Hashimoto $\mathrm{T}$. Is closed-suction drainage necessary for single-level lumbar decompression?: review of 560
cases.Clin Orthop Relat Res 2010;468:2690-4.

13. Sokolowski MJ, Garvey TA, Perl J 2nd, et al. Prospective study of postoperative lumbar epidural hematoma: incidence and risk factors. Spine (Phila Pa 1976) 2008;33:108-13.

14. Pospiech J, Pajonk F, Stolke D. Epidural scar tissue formation after spinal surgery: an experimental study. Eur Spine J 1995;4:213-9.

15. Abitbol JJ, Lincoln TL, Lind BI, Amiel D, Akeson WH, Garfin SR. Preventing postlaminectomy adhesion: a new experimental model. Spine (Phila Pa 1976) 1994;19:1809-14. 